\title{
RASSF2 hypermethylation is present and related to shorter survival in squamous cervical cancer
}

\author{
David Guerrero-Setas ${ }^{1}$, Noemí Pérez-Janices ${ }^{1}$, Laura Blanco-Fernandez ${ }^{1}$, Amaya Ojer ${ }^{1}$, \\ Koldo Cambra ${ }^{1}$, María Berdasco ${ }^{2}$, Manel Esteller ${ }^{2}$, Sergio Maria-Ruiz ${ }^{1}$, Natalia Torrea ${ }^{1}$ and \\ Rosa Guarch ${ }^{3}$ \\ ${ }^{1}$ Cancer Epigenetics Group, Navarrabiomed, Miguel Servet Foundation, Pamplona, Navarra, Spain; ${ }^{2}$ Cancer \\ Epigenetics Group, Cancer Epigenetics and Biology Programme (PEBC), Bellvitge Biomedical Research \\ Institute (IDIBELL), Barcelona, Spain and ${ }^{3}$ Department of Pathology (Section B-Hospital Virgen del Camino), \\ Hospital Complex of Navarra, Navarra Health Service, Pamplona, Navarra, Spain
}

\begin{abstract}
Ras association (RalGDS/AF-6) domain family member 2 (RASSF2) is a gene involved in the progression of several human cancers, including breast, colorectal and lung cancer. The aims of this study were to determine the hypermethylation of the gene in squamous cervical cancer and precursor lesions, along with that of RASSF1 and the recently described EPB41L3, and to analyze the potential prognostic role of these genes. Methylation-specific PCR and bisulfite sequencing were used to analyze the methylation status of RASSF2 and EPB41L3 gene in 60 squamous cervical cancer, 76 cervical intraepithelial neoplasias grade III, 16 grade II, 14 grade I and 13 cases of normal tissue adjacent to cervical intraepithelial neoplasia. RASSF2 expression was evaluated by immunohistochemistry and the re-expression of RASSF2 and EPB41L3 was analyzed by quantitative reverse-transcription PCR in HeLa, SiHa, C33A and A431 cell lines treated with 5-aza-2'deoxycytidine and/or trichostatin. RASSF1 hypermethylation and human papillomavirus type were also analyzed in all the cases by methylation-specific PCR and reverse line blot, respectively. RASSF2 hypermethylation was predominant in squamous cervical cancer $(60.9 \%)$ compared with cervical intraepithelial neoplasias (4.2\%) and was associated with a lower level of RASSF2 expression and vascular invasion in squamous cervical cancer. EPB41L3 and RASSF1 hypermethylations were also more frequent in cancer than in precursor lesions. Patients with RASSF2 hypermethylation had shorter survival time, independent of tumor stage (hazard ratio: 6.0; 95\% confidence interval: 1.5-24.5). Finally, the expressions of RASSF2 and EPB41L3 were restored in several cell lines treated with 5-aza-2'-deoxycytidine. Taken together, our results suggest that RASSF2 potentially functions as a new tumor-suppressor gene that is inactivated through hypermethylation in cervical cancer and is related to the bad prognosis of these patients.
\end{abstract}

Modern Pathology (2013) 26, 1111-1122; doi:10.1038/modpathol.2013.32; published online 29 March 2013

Keywords: 5-aza-2'-deoxycytidine; cervical cancer; DNA methylation; EPB41L3; prognosis; RASSF1; RASSF2

Cervical cancer is the third most common cause of cancer deaths in women worldwide; ${ }^{1}$ the major etiological factor is the presence of human papillomavirus (HPV), with HPV 16, 18, 31, 33, 35, 45,52 and 58 being the most prevalent types. ${ }^{2}$ Infection with high-risk HPV is the main risk factor for developing high-grade cervical intraepithelial neoplasia grade II (moderate dysplasia) and grade III

Correspondence: Dr D Guerrero-Setas, PhD, Biomedical Research Center, Navarra Health Service, Miguel Servet Foundation, 3 Irunlarrea Street, Pamplona, Navarra 31008, Spain.

E-mail: dguerres@navarra.es

Received 25 October 2012; revised 15 January 2013; accepted 16 January 2013; published online 29 March 2013 (severe dysplasia), which are precursor lesions of squamous cervical carcinomas. $^{3}$ The risk of developing squamous cervical carcinomas depends not only on HPV infection and persistence ${ }^{4}$ but also on the presence of molecular alterations, including the hypermethylation of the CpG islands of tumorsuppressor genes. ${ }^{5}$ These epigenetic abnormalities in cancer can affect a plethora of genes involved in key cellular pathways, including cell cycle control and cell adhesion. ${ }^{5}$

Ras association (RalGDS/AF-6) domain family member 2 (RASSF2) is a gene located at 20p13, with RASSF2A as the most important putative transcript. ${ }^{6}$ It belongs to the RAS association domain family of proteins, which has important functions in cell-cycle 
control, microtubule stabilization and motility. ${ }^{7}$ The RASSF2A protein acts as a negative effector of K-RAS protein via the Ras effector domain, ${ }^{8}$ and it relocates to the nucleus, where it performs important apoptotic functions. ${ }^{9}$ RASSF2 is inactivated by hypermethylation in breast, colorectal, endometrial, gastric, lung and thyroid cancers, ${ }^{9-13}$ but the presence of this alteration in cervical cancer and precursor lesions and its prognostic role has not yet been analyzed. In this study, apart from RASSF2, we have also included the analysis of the hypermethylation in premalignant lesions and cervical cancer of $R A S S F 1$ from the same family as $R A S S F 2$, and $E P B 41 L 3$, a gene recently found to be hypermethylated in cervical cancer.

The RASSF1 gene shares important functions with RASSF2 in Ras signaling and microtubule stabilization, ${ }^{14}$ and is located at $3 p 21$, where it is frequently deleted or hypermethylated in cervical cancer ${ }^{11}$ and cervical intraepithelial neoplasia. ${ }^{15}$ Its hypermethylation is of prognostic significance in association with RASSF2 hypermethylation in oral squamous cell carcinoma. ${ }^{16}$ On the other hand, EPB41L3 hypermethylation has recently been described in cervical cancer, ${ }^{17}$ but its prognostic role and involvement in precursor lesions are not known. This gene codes for a crucial protein in cell adhesion and is inactivated in several tumor types, such as breast tumors and renal cell carcinomas, ${ }^{18,19}$ showing a bad prognosis role in non-small-cell lung cancer. ${ }^{20}$

Our objectives in this study were to analyze the methylation status of RASSF2, RASSF1 and $E P B 41 L 3$ in cervical intraepithelial neoplasia and squamous cervical carcinomas, and to analyze their prognostic role. The treatment of HPV-positive and HPV-negative cell lines with the demethylating agent 5-aza-2'-deoxycytidine (dC) and/or the histone deacetylase inhibitor trichostatin A was also studied to examine the promoter demethylation and the restoration of the mRNA expression of the less studied EPB41L3 and RASSF2 genes.

\section{Materials and methods}

\section{Cell Lines}

HPV-positive cervical cell lines HeLa (HPV 18) and SiHa (HPV 16) and HPV-negative cervical cell line C33A and vulvar cell line A431 were included in this study. These cells were grown in Dulbecco's modified Eagle's medium supplemented with 10\% fetal bovine serum (Invitrogen, Life Technologies, Carlsbad, CA, USA), and $1 \%$ penicillin/streptomycin (Invitrogen, Life Technologies) in a humidified atmosphere (5\% $\mathrm{CO}_{2}, 37^{\circ} \mathrm{C}$ ) until $80 \%$ confluence was attained.

\section{Patients}

The group of cases studied consisted of patients diagnosed with squamous cervical carcinomas
(60 patients) and cervical intraepithelial neoplasia III lesions (76 patients) at Hospital Virgen del Camino (Navarra Hospital Complex, Navarra Health Service, Pamplona, Spain) between January 1995 and December 2006 (Table 1 and Figures 1a and b). In all, 14 and 16 cervical intraepithelial neoplasia I and II, respectively, and 13 cases of normal tissue adjacent to cervical intraepithelial neoplasia I, all randomly selected from the same period, were also analyzed. None of the patients had received radiation or chemotherapy before surgery. The study was approved by the Clinical Research Ethics Committee of Navarra.

All the cervical intraepithelial neoplasia lesions had been resected by loop electrosurgical excision procedure; 48 of 60 patients with squamous cervical carcinomas underwent primary surgical resection (total hysterectomy with bilateral salpingo-oophorectomy and lymphadenectomy), and 12 patients were diagnosed by biopsy on the basis of the patient's age and health status, and staged by radiology and lymphadenectomy. The diagnosis of cervical intraepithelial neoplasia lesions and squamous cervical carcinomas was confirmed following microscopic inspection by a gynecological pathologist.

The pathological staging of resected tumors according to the Federation Internationale de Gynécologie et d'Obstétrique (FIGO) was known for 44 out of 48 cases with primary surgical resection ${ }^{3}$ (Table 1). Out of 19 stage I squamous cervical carcinomas, 6 were microinvasive carcinomas (early invasive squamous cell carcinoma), characterized by early stromal invasion according to previously

Table 1 Age of cervical intraepithelial neoplasia and squamous cervical carcinoma patients, and pathological data of resected squamous cervical tumors

\begin{tabular}{lr}
\hline Clinical data & \\
Mean age (s.d.) & \\
Cervical intraepithelial neoplasia & 34 (9.0) years \\
I & $35(8.5)$ years \\
II & $39(10)$ years \\
III & $55(13)$ years \\
Squamous cervical carcinoma & \\
Pathological data & \\
Mean tumor size (s.d.) (cm) & $2.7(1.8)$ \\
FIGO stage & $44(100 \%)$ \\
I & $19(43 \%)$ \\
II & $8(18 \%)$ \\
III & $12(27 \%)$ \\
IV & $5(11 \%)$ \\
Grade of differentiation ${ }^{\mathrm{a}}$ & $48(100 \%)$ \\
Low & $15(31 \%)$ \\
Intermediate & $25(52 \%)$ \\
High & $8(17 \%)$ \\
Vascular invasion & $41(100 \%)$ \\
Yes & $25(61 \%)$ \\
No & $16(39 \%)$ \\
Histological type & $48(100 \%)$ \\
Microinvasive & $6(12.5 \%)$ \\
Squamous & $42(87.5 \%)$ \\
\end{tabular}

\footnotetext{
${ }^{\mathrm{a} A c c o r d i n g}$ to the World Health Organization grading system.
} 

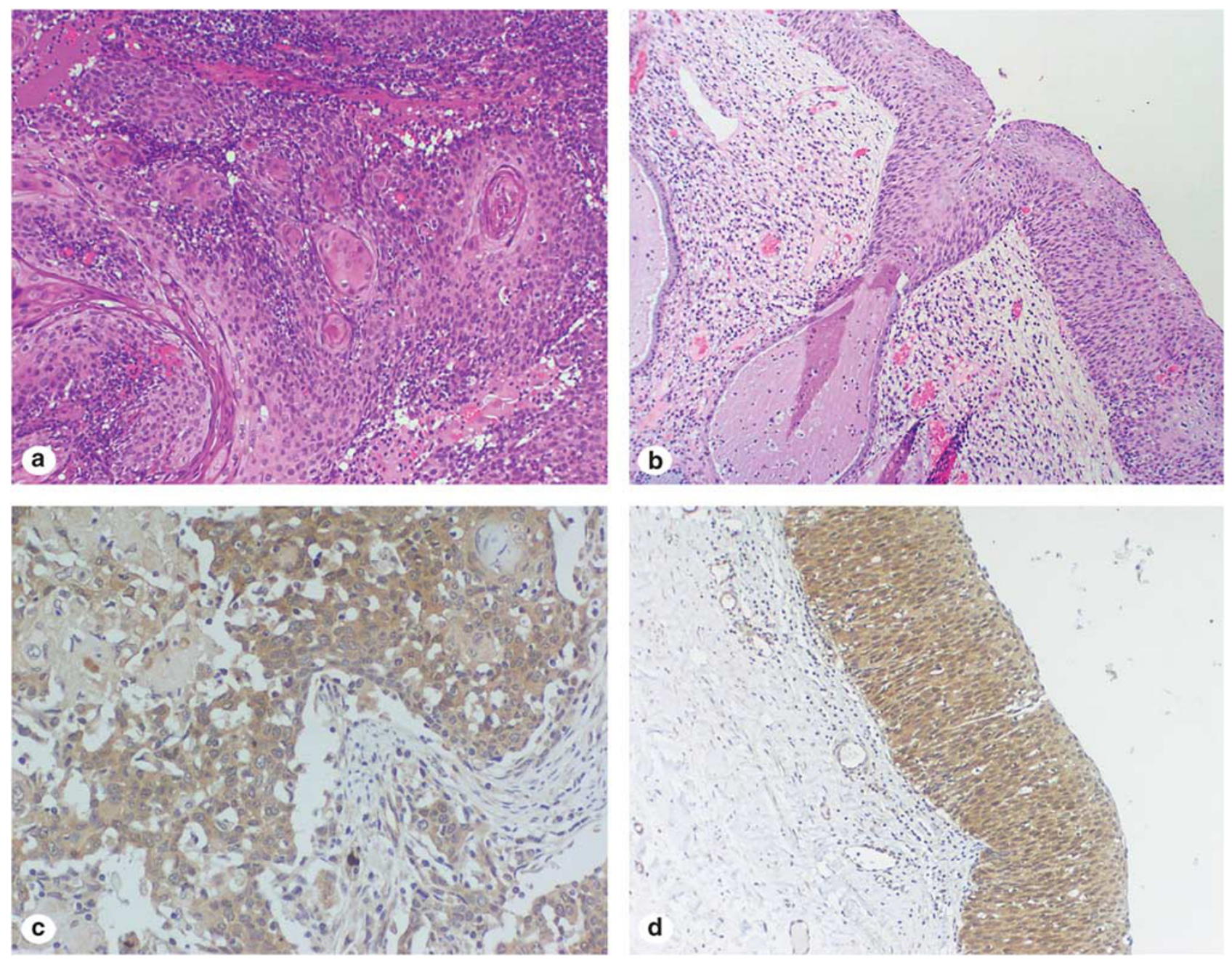

Figure 1 (a) Detail of a squamous cervical carcinoma (hematoxylin and eosin (H\&E), $\times 400$ ). (b) Panoramic view of cervical intraepithelial neoplasia grade III lesion with the loss of maturation of the epithelium (H\&E, $\times 100)$. (c) Immunohistochemistry staining for a tumor positive (including positive endothelial cells as internal control) for RASSF2 expression, corresponding to an unmethylated case $(\times 400)$. (d) Immunohistochemistry staining for a cervical intraepithelial neoplasia III, positive for RASSF2 $(\times 100)$.

reported criteria. $^{3}$ Additional pathological data available for these cases are shown in Table 1.

The oncological treatment of the patients with advanced stages (II, III and IV) (56.9\%) was complemented by radiotherapy, according to standard protocols. ${ }^{21}$ The follow-up included physical and clinical examination, and a pelvic computed tomography scan and radiography every 3 months for the first 2 years, and every 6 months thereafter.

\section{DNA Extraction from Cases}

The pathologist selected a representative area of the lesions (cervical intraepithelial neoplasia, tumors) and normal tissue on formalin-fixed, paraffin-embedded sections stained with hematoxylin and eosin. The selected tissue was scraped from $5-\mu \mathrm{m}-$ thick tissue sections under microscopic guidance, then deparaffinized in xylene and digested overnight at $56^{\circ} \mathrm{C}$ in $50-400 \mu \mathrm{l}$ buffer containing proteinase $\mathrm{K}(10 \mathrm{mg} / \mathrm{ml})$ (Roche, Indianapolis, IN, USA), depending on the quantity of tissue. After inactivation of proteinase $\mathrm{K}$ at $96^{\circ} \mathrm{C}$ for $10 \mathrm{~min}$, DNA concentration and quality was measured for each sample using the NanoDrop spectrophotometer (ND-1000; NanoDrop Technologies, Wilmington, DE, USA). To assess DNA quality, the $\beta$-globin gene was amplified by PCR using primers BGPCO3 and BGPCO5, which generated a 209-bp product. $^{22}$

\section{Detection and Genotyping of HPV in Cases}

We used PCR linked to a non-radioactive reverse-line blot procedure. ${ }^{23}$ This technique amplifies a 150-bp fragment of viral DNA by adding GP5 +/GP6+ biotinylated primers. HPV genotypes were determined by hybridizing the GP5 +/6+ PCR products with specific oligoprobes containing a 
5'-amino group on a carboxyl-coated nylon membrane (Biodyne C; Pall Corp., East Hills, NY, USA) in a miniblotter (MN45; Immunetics, Boston, MA, USA). The membrane was then incubated with streptavidin-POD conjugate (Roche, Indianapolis, IN, USA) subjected to enhanced chemiluminescence (ECL; GE Healthcare, Munich, Germany) and scanned in a densitometer (BioRad Laboratories, Hercules, CA, USA). A total of 36 types were identified using oligoprobes against high-risk types (HPV 16, 18, 31, $33,35,39,45,51,52,56,58,59,68,73,82 / \mathrm{MM} 4$ and 82/IS39), probable high-risk types (HPV 26, 53 and 66) and low-risk types (HPV 6, 11, 34, 40, 42, 43, 44, 54, 55, 57, 61, 70, 71/ CP8061, 72, 81/CP83104, 83/ MM7, 84/MM8 and CP6108). A case positive for HPV 16 and distilled water were included as positive and negative controls, respectively.

\section{Methylation-Specific PCR in Cases}

The methylation status of RASSF2, RASSF1 and $E P B 41 L 3$ genes was determined in all the cases after sodium bisulfite modification of $1 \mu \mathrm{g}$ of genomic DNA. ${ }^{24}$ This treatment modifies unmethylated, but not methylated, cytosine to uracil and the modified DNA can be amplified with specific primers capable of annealing specifically methylated and unmethylated sequences. The primer sequences and the annealing temperatures (Sigma, St Louis, MO, USA) used to study RASSF1 and RASSF2 genes (Supplementary Table 1) have been described previously. ${ }^{25}$ Primers used to amplify a region located -141 to $-30 \mathrm{bp}$ from the transcription start point of EPB41L3 were designed using Methyl Primer Express v.1.0 (Applied Biosystems, Foster City, CA, USA) (Supplementary Table 1). Positive and negative controls were included in each bisulfite treatment and methylation-specific PCR to confirm the presence or absence of the band of expected size for each control, respectively. DNA from lymphocytes treated in vitro with SssI methylase (New England Biolabs, Ipswich, MA, USA) was used as the positive control for methylated alleles of these genes. DNA from normal lymphocytes was used as the negative control. The presence of PCR products was verified by $2 \%$ agarose gel electrophoresis, stained with ethidium bromide and examined under ultraviolet illumination. Gene hypermethylation was defined as the presence of the band of expected size in the methylation reaction. The case was considered not analyzable if the gel band was not present in any of the two reactions (methylated and unmethylated).

\section{5-AzadC and Trichostatin Treatment in Cell Lines}

To determine the effects of DNA demethylation and acetylation on the restoration of mRNA expression of the two less-studied genes, RASSF2 and EPB41L3, cell lines were cultured and treated with 5-azadC and/or trichostatin. A total of $10^{6}$ cells were seeded and allowed to grow for $24 \mathrm{~h}$ before the treatment. Cells were treated separately with $10 \mu \mathrm{g} / \mathrm{ml} 5$-azadC (Sigma), diluted in phosphate-buffered saline (PBS) or PBS alone for $96 \mathrm{~h}$, and with $200 \mathrm{mg} / \mathrm{ml}$ trichostatin (Sigma) diluted in PBS or PBS alone for $24 \mathrm{~h}$, or with both treatments (5-azadC for $96 \mathrm{~h}$ and trichostatin for the final $24 \mathrm{~h}$ ). The medium containing the drug was replaced every $24 \mathrm{~h}$. Cells receiving 5-azadC and combination treatment were trypsinized and harvested after $96 \mathrm{~h}$, and cells in trichostatin treatment were collected after $24 \mathrm{~h}$ of treatment.

DNA and RNA were obtained for controls and for 5-azadC and/or trichostatin-treated cells using the EZNA Tissue DNA kit (Omega Bio-Tek, Norcross, GA, USA) and Ribopure Kit including a DNAse I treatment (Ambion, Life Technologies, Carlsbad, CA, USA), respectively, according to the manufacturer's instructions.

\section{Quantitative Reverse Transcription-PCR for RASSF2 and EPB41L3 Expression in Cell Lines}

Quantitative reverse-transcription PCR was performed to analyze the level of mRNA expression of RASSF2 and EPB41L3 genes in control and treated cell lines. cDNA was synthesized from $1 \mu \mathrm{g}$ of RNA in a total volume of $50 \mu \mathrm{l}$ with $250 \mathrm{U}$ of reverse transcriptase, $2 \times$ reverse transcriptase buffer, $1 \mu \mathrm{M}$ random primers and $8 \mathrm{mM}$ dNTPs, using a reverse transcription kit (Life Technologies).

Real-time PCR reactions were performed in triplicate from $100 \mathrm{ng}$ of cDNA, for each control and treated cell line, together with positive and negative controls, using the Applied Biosystems 7300 Sequence Detection System (Life Technologies). Each real-time PCR reaction consisted of $50 \mu \mathrm{l}$ of reaction mixture containing $25 \mu \mathrm{l}$ PCR Master Mix (TaqMan Universal PCR Master Mix; Applied Biosystems) and $1 \times$ TaqMan gene expression assays for target (RASSF2; Assay Id: Hs00248129_m1 (Applied Biosystems); EPB41L3; Assay Id: Hs00202360_m1) and endogenous control (GAPDH; Applied Biosystems) genes. The reaction consisted of $50{ }^{\circ} \mathrm{C}$ for 2 min (AmpErase UNG activation), followed by $95^{\circ} \mathrm{C}$ for $10 \mathrm{~min}$ (AmpliTaq Gold activation) and 40 cycles of PCR amplification $\left(95^{\circ} \mathrm{C}\right.$ for $15 \mathrm{~s}$ and $60^{\circ} \mathrm{C}$ for $1 \mathrm{~min}$ ). The cycle threshold value (Ct value) of each gene was normalized to that of housekeeping GAPDH obtained from the same sample. The deltadelta Ct method was used to calculate the relative fold-change in the expression of genes of each treatment relative to the control value.

\section{Bisulfite Sequencing of RASSF2 and EPB41L3 Genes in Cases and Cell Lines}

Bisulfite sequencing was performed to analyze RASSF2 hypermethylation, which has not been described previously in cervical lesions, and 
EPB41L3 hypermethylation, which has only recently been reported in cervical cancer. ${ }^{17}$ This analysis was performed in cervical intrapithelial lesions, tumors and the HPV-positive (HeLa) and HPV-negative (A431) cell lines treated with 5-azadC or trichostatin, alone or in combination.

The analysis involved the use of 10 clones obtained from one case of normal tissue, cervical intraepithelial neoplasia I-III and tumor, following bisulfite conversion and amplification of a fragment containing $\mathrm{CpG}$ islands by PCR covering the same region as BS. Specific primers were designed using Methyl Primer Express v.1.0 (Applied Biosystems); sequences and conditions are described in Supplementary Table 1. PCR products were gelpurified and cloned into the pGEMT Easy Vector System (Promega, Fitchburg, WI, USA) according to the manufacturer's protocol. In all, 10 colonies were randomly chosen, and DNA was extracted and sequenced using the 3100 Genetic Analyzer (Applied Biosystems). The results were transformed into percentages of CpGs showing methylation.

\section{Immunohistochemistry}

The 4- $\mu$ m-thick sections from 20 cervical intraepithelial neoplasia and 20 tumors randomly selected (methylated and unmethylated cases) were mounted on glass slides, deparaffinized and rehydrated. Briefly, antigen retrieval was performed using Bond Epitope Retrieval Solution 2 (pH 9.0) (cat. no. AR9640; A Menarini Diagnostics Ltd, Berkshire, UK) at $95{ }^{\circ} \mathrm{C}$ for $30 \mathrm{~min}$. Slides were then incubated with rabbit monoclonal RASSF2 antibody (clone EPR6621; Epitomics, San Diego, CA, USA) diluted 1:100 for $60 \mathrm{~min}$ at room temperature. The primary antibody was developed using Bond Polymer Refine Detection (cat. no. DS9800; Menarini Diagnostics Ltd) and visualized with $3,3^{\prime}$-diaminobenzidine. Finally, slides were counterstained with hematoxylin. RASSF2 staining was expressed diffusely in the cytoplasm and, in some cases, in the nucleus. The extent and intensity of immunopositivity were both considered, and evaluation of immunohistochemistry was independently evaluated by two pathologists, who scored the cases as positive or weakly negative, on the basis of the previous criteria. ${ }^{26}$

\section{Statistical Analysis}

Associations between gene hypermethylation, pathological and clinical variables of this retrospective study were assessed with the $\chi^{2}$ test or Fisher's exact test. Disease-free survival was studied in patients with squamous cervical carcinomas undergoing primary surgical resection $(n=48)$ and overall survival in all patients with squamous cervical cancer $(n=60)$.
Survival curves were calculated using the Kaplan-Meier method and statistically compared by the log-rank test. Cox proportional hazard regression models were used for univariate analysis and the proportional hazard ratio and the 95\% confidence intervals (95\% CI) were calculated for each factor. Hazard risk was adjusted for tumor stage and patient age. Statistical significance was concluded for values of $P<0.05$ in all cases.

\section{Results}

\section{HPV Typing}

HPV was present in $93.4 \%$ and $94.9 \%$ of cervical intraepithelial neoplasia III and squamous cervical carcinomas, respectively (Supplementary Figure 1). The percentages were lower in the case of cervical intraepithelial neoplasia I (64.3\%) and II (75\%) lesions. All the HPV-positive lesions contained high-risk HPV types, with HPV 16 and HPV 18 being the most prevalent, alone or in combination with other high-risk types (Supplementary Table 2).

\section{Methylation-Specific PCR Results}

There were different numbers of analyzable cases for the RASSF2, RASSF1 and EPB41L3 genes (Table 2, Supplementary Table 3 and Figure 2). The percentages of methylation were higher for all the genes in tumors $(65.5 \%, 46.3 \%, 66.1 \%$ for RASSF $2, R A S S F 1$ and $E P B 41 L 3$, respectively) than in cervical intraepithelial neoplasia III $(4.2 \%, 11.8 \%$ and $32.4 \%$, respectively), the differences being statistically significant for all the genes (Table 2). On the other hand, cervical intraepithelial neoplasia I and II cases were slightly methylated for all the genes except for $R A S S F 2$, which was not methylated in any cervical intraepithelial neoplasia I-II case (Supplementary Table 3). Normal tissue was not methylated for any of the genes.

It is notable that the proportion of RASSF2 hypermethylation was much greater among squamous cervical carcinomas (38 of 58 analyzable cases) than among cervical intraepithelial neoplasia III lesions (3 of 71 analyzable cases) $(P<0.001)$ (Table 2). In addition, there was a clear relationship between RASSF2 gene hypermethylation and vascular invasion $(P=0.033)$ (Table 2$)$. All the patients who showed metastasis at the time of diagnosis exhibited hypermethylation of the RASSF2 gene (five patients), but there was no association between this alteration and lymph node involvement.

In univariate analyses, RASSF2 and EPB41L3 hypermethylations were both significantly associated with HPV presence $(P=0.004$ and 0.034 , respectively). Nevertheless, these associations were not significant when adjusting for disease category in logistic models (Supplementary Table 4). Finally, 
Table 2 Association of gene hypermethylation with pathological diagnosis, vascular invasion and histological type

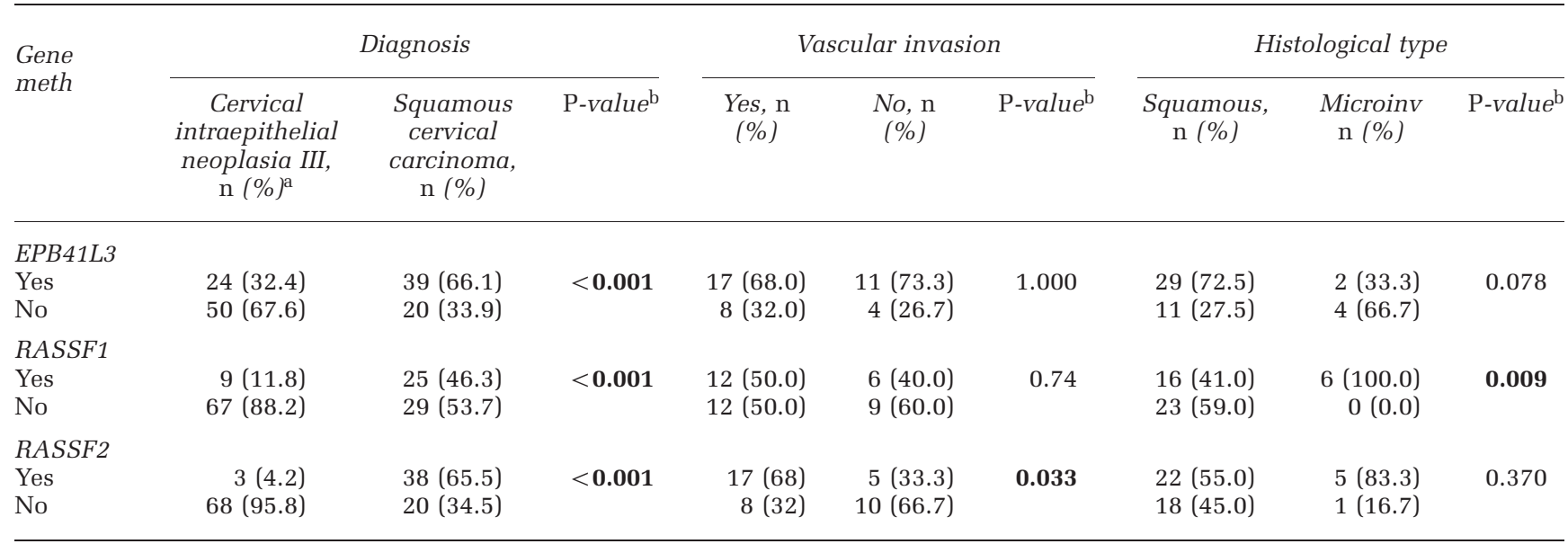

Abbreviations: meth, methylation; microinv, microinvasive type.

${ }^{a}$ Number and percentage of cases with available data.

$\mathrm{b} \chi^{2}$-test $P$-values (statistically significant $P$-values in bold).

RASSF2 and RASSF1 hypermethylations were not associated with one another.

\section{BS Results for RASSF2 and EPB41L3 Genes}

In the case of RASSF2, promoter hypermethylation was confirmed to be preferentially present in tumors compared with cervical intraepithelial neoplasia III (Figure 3, up). On the other hand, cervical intraepithelial neoplasia II had a low level of RASSF2 hypermethylation (methylation in $16.7 \%$ of the CpGs analyzed) and normal cervical tissue and cervical intraepithelial neoplasia I revealed unmethylated RASSF2 for all the clones analyzed.

In the case of $E P B 41 L 3$, bisulfite sequencing also enabled us to confirm the increasing hypermethylation of this gene from cervical intraepithelial neoplasia to tumor, with CpG methylation in $15.9 \%, 26.3 \%$ and $68.8 \%$ of the clones, respectively (Figure 3, down).

\section{Quantitative Reverse Transcription-PCR Results for the RASSF2 and EPB41L3 Genes}

In the case of RASSF2, quantitative reverse-transcription PCR confirmed that treatment with trichostatin led to partial re-expression of RASSF2 in HeLa cells $(P=0.045)$ (Figure 4 , up). In C33A cells, the treatment with 5-azadC alone led to higher levels of RASSF2, especially when combined with trichostatin $(P=0.040)$. The effect of these agents was also analyzed in A431 cells, which showed prominent, approximately 11-fold $(P=0.001)$ and 15-fold $(P=0.001), R A S S F 2$ re-expression, respectively.

In the case of EPB41L3, 5-azadC treatment was sufficient to boost its expression only in the SiHa cell line, treated with this agent alone or in combination with trichostatin $(P=0.040)$. Conver- sely, gene re-expression was absent from HeLa, C33A and A431 cell lines (Figure 4, down).

\section{RASSF2 Immunohistochemistry Results}

In squamous cervical carcinomas, expression was diffuse in the cytoplasm and present in some isolated nuclei in all the cases with unmethylated promoter (Figure 1c). Conversely, RASSF2 staining was characterized as being reduced or absent in the majority of cases with methylated promoter $(P=0.001)$. Endothelial cells were clearly stained and were used as the positive internal control, in cases that showed weak or no expression. On the other hand, all the cervical intraepithelial neoplasia cases and normal tissue stained intensely positive for this protein (Figure 1d).

\section{Follow-Up Results}

Disease-free survival and overall survival were shorter for patients with more advanced stages of squamous cervical carcinomas and vascular invasion at the time of diagnosis (Table 3). It is notable that overall survival was significantly shorter for patients with $R A S S F 2$ hypermethylation $(P=0.019$; Table 3 and Figure 5). After adjusting for age and tumor stage, the risk of recurrence remained higher in patients with RASSF2 hypermethylation (hazard ratio $=6.2 ; 95 \%$ CI: 1.2-32.4) (Table 4). Risk estimates related to mortality in all the patients were similar, with a hazard ratio for RASSF2 hypermethylation of 6.0 (CI: 1.5-24.5).

\section{Discussion}

The analysis of markers and their prognostic role in cervical cancer, such as the epigenetic analysis of 
RASSF2

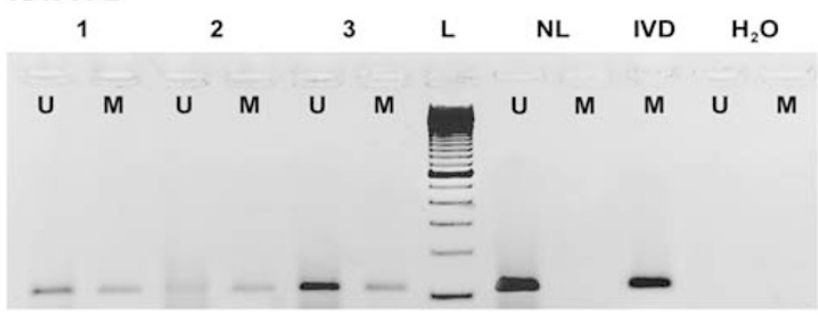

EPB41L3

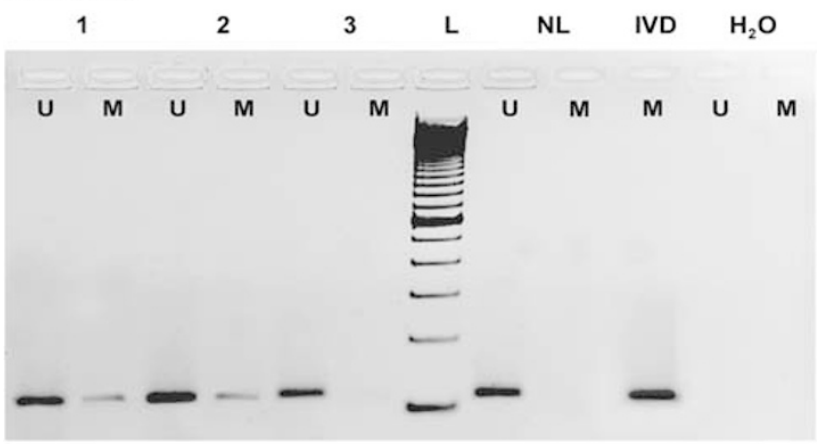

RASSF1

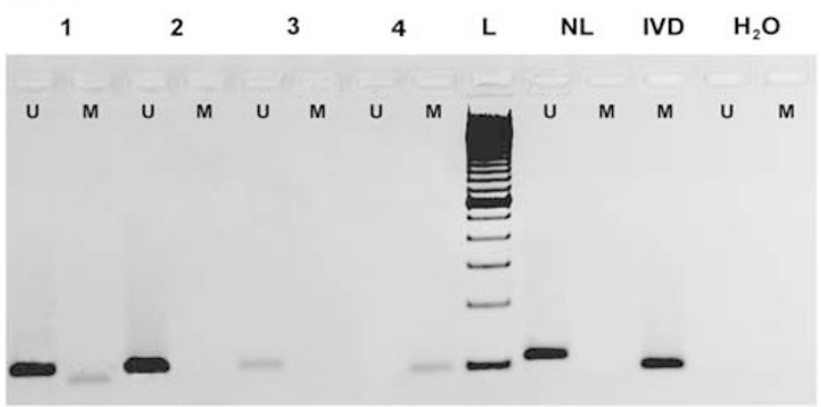

Figure 2 Methylation-specific RASSF2, EPB41L3 and RASSF1 genes. Both the unmethylated (U) and methylated (M) PCR products are shown for each case. DNA from normal lymphocytes (NL) and in vitro-methylated DNA (IVD) are used as a positive PCR control for the $U$ and $M$ reactions, respectively; $\mathrm{H}_{2} \mathrm{O}$ : negative control.

tumor-suppressor genes, is crucial for detecting more aggressive squamous cervical carcinomas that are characterized by their lethality. ${ }^{27}$ Gene hypermethylation has been described in various stages of HPV 16/18-transfected keratinocytes, ${ }^{28}$ and is preferentially present in repetitive DNA sequences $^{29}$ and specific genes. ${ }^{30}$ These epigenetic alterations are involved in the pathogenesis and progression of cervical carcinoma from cervical intraepithelial neoplasia to invasive tumors, as was reported for MGMT and $p 16$ genes, among others. ${ }^{15,31}$

This association between the methylation changes and malignancy has been found in our squamous tumors, in which all the genes were more methylated than in cervical intraepithelial neoplasia III cases. This difference is remarkable in the case of $R A S S F 2$, as this gene is almost exclusively hypermethylated in tumors $(65.5 \%)$; it is found in a very small proportion of cervical intraepithelial neoplasia III $(4.2 \%)$. The value of RASSF2 hypermethylation as a tumor marker has already been noted in patients with gastric, ${ }^{9}$ colorectal, ${ }^{32}$ non-small-cell lung cancer and breast cancer. ${ }^{10}$

In our study, the upregulation of RASSF2 mRNA expression was much higher in HPV-negative cell lines treated by 5 -azadC treatment and in HPVpositive cells treated by trichostatin. These results suggest that histone deacetylation contributes to this silencing in HPV-positive cell lines probably caused by the virus, as was previously suggested for the reexpression of the cystatin gene after trichostatin treatment in SiHa cells. ${ }^{33}$ The involvement of the RASSF2 gene in RAS signaling, ${ }^{34}$ and the promotion of apoptosis and ras-dependent cell growth inhibition demonstrated in pancreatic cancer cells and breast cancer cells transfected with the RASSF2 gene ${ }^{10,35}$ highlight the need for further studies of the role of this gene in cervical cancer cell lines.

We note that the presence of RASSF2 hypermethylation in gynecological cancer in comparison with benign lesions was first reported by our group in vulvar squamous cell carcinoma. ${ }^{36}$ To our knowledge, this is the first description of the same alteration in a high proportion of squamous cervical carcinomas, correlated with the loss of expression of the RASSF2 protein detected by immunohistochemistry. RASSF2 hypermethylation is equally common in tumor stages I-IV of squamous cervical carcinomas, suggesting that it is an early event in cancer progression, as was described for this gene in colorectal cancer, and is equally hypermethylated in stages I and II. ${ }^{32}$ In addition, its possible oncogenic role is reflected in its association with vascular invasion, which is a bad prognostic factor for our patients. ${ }^{37}$ It should also be stressed that RASSF2 hypermethylation is a bad prognostic factor, with respect to overall survival, in our patients. The bad prognostic role of this gene is also characterized by radioresistance and shorter survival in oral squamous carcinoma, and pancreatic and gastric cancers, in which it is more frequent in cases with peritoneal recurrence. ${ }^{16,38-40}$

On the other hand, in our study, RASSF1 hypermethylation was also more frequent in squamous cervical carcinomas than in cervical intraepithelial neoplasia III and was not found to be of prognostic significance, as reported previously. ${ }^{41,42}$ This epigenetic alteration was also present in the six microinvasive cancers examined here, suggesting that this gene is involved in early tumor invasion, as was described for CCNA1 and C13ORF18 hypermethylations in high-grade cervical lesions and microinvasive cervical cancer. ${ }^{43}$ In fact, a similar finding was described for the clear association between RASSF1 hypermethylation and microinvasion in ovarian borderline tumors. ${ }^{44}$ More studies with a wide range of microinvasive populations in cervical cancer are needed to confirm this finding. 


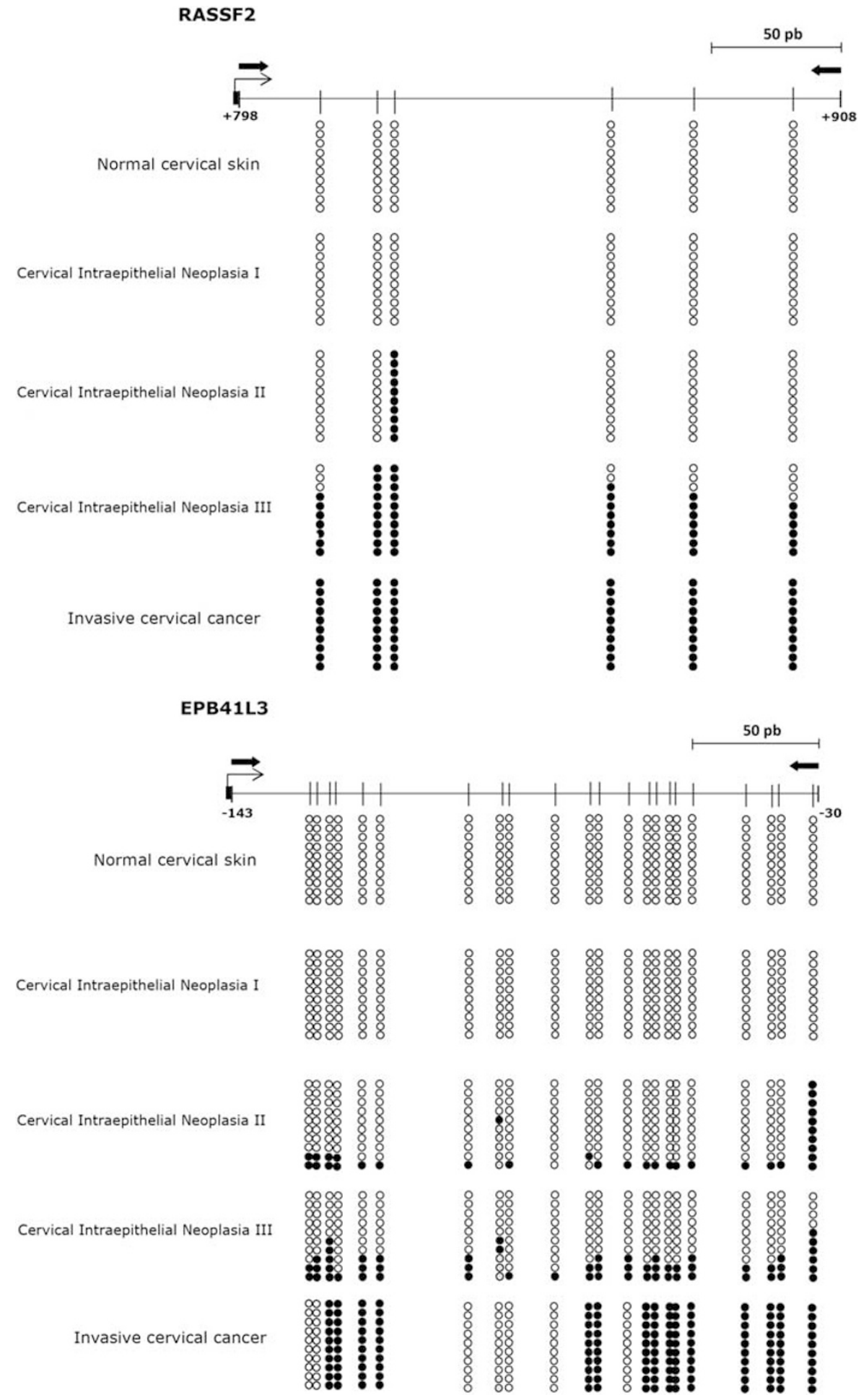

Figure 3 Bisulfite sequencing of RASSF2 (up) and EPB41L3 (down) genes in clones derived from normal, cervical intraepithelial neoplasia I-III lesions and tumors. Each vertical bar of circles represents a CpG site in the promoter region and the methylation status of the corresponding site is indicated in the circle below. Open and filled circles represent unmethylated and methylated CpG sites, respectively, and each row represents a single clone.

Finally, the predominance of this alteration in squamous cervical carcinomas compared with precursor lesions has also been found for the EPB41L3 gene, which is hypermethylated in cervical cancer. ${ }^{17,45}$ In non-cancerous cells, EPB41L3 protein anchors to the actin cytoskeleton at the cell-cell attachment site, crucial to the adhesion of adjacent cells. ${ }^{45}$ Additional functions of EPB41L3 in relation 

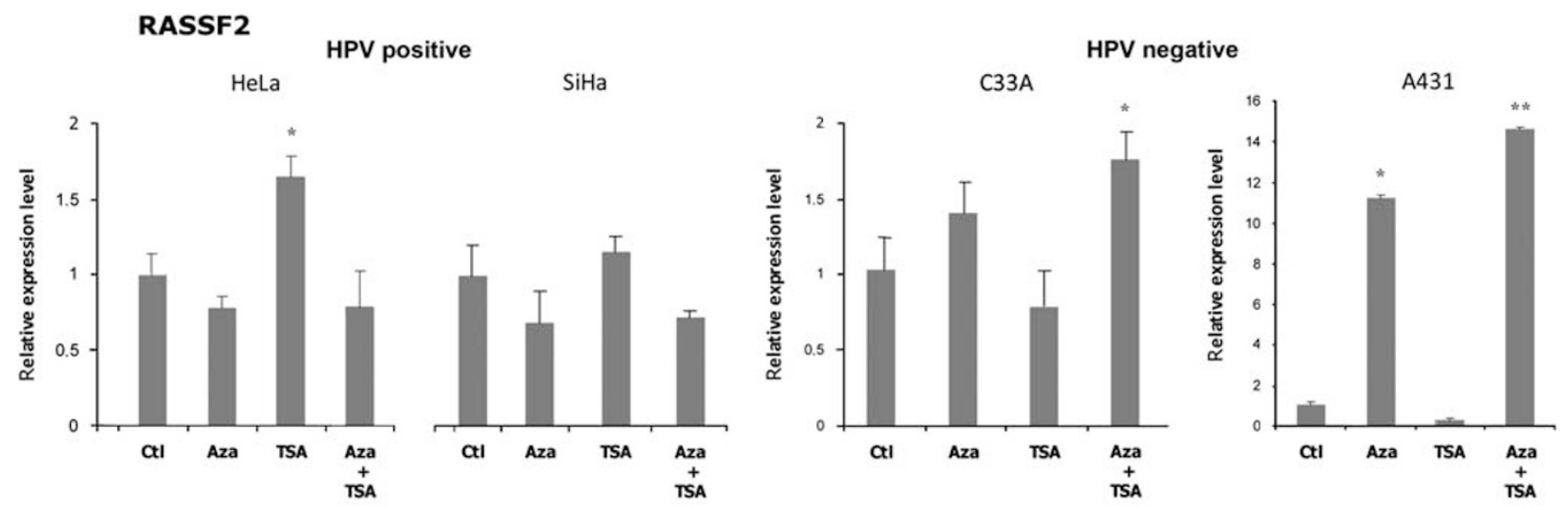

\section{EPB41L3}
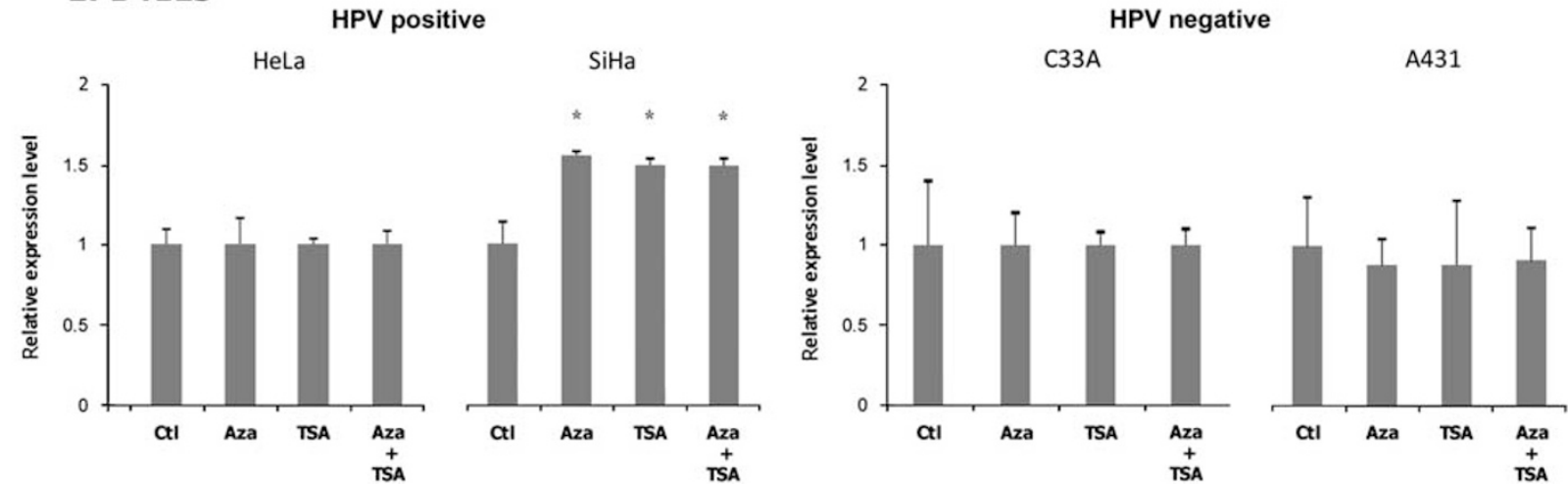

Figure 4 Expression level of RASSF2 (up) and EPB41L3 (down) in human papillomavirus (HPV)-positive and HPV-negative cells treated with 5-aza-2'-deoxycytidine and/or trichostatin. Relative level of expression after quantitative real-time PCR (qRT-PCR) was compared with the untreated cells (control, Ctl), whose value was taken as $100 \%$. Bars represent mean \pm s.d. of the relative expression level of three experiments. Statistically significant $\left({ }^{*} P<0.05\right)$ and very significant $\left({ }^{*} P<0.01\right)$ change in gene expression in treated compared with untreated cells.

Table 3 Univariate Kaplan-Meier analysis of the risk of recurrence or death related to pathological and molecular variables in patients with squamous cervical cancer

\begin{tabular}{|c|c|c|c|c|c|c|c|c|c|c|}
\hline \multirow[t]{3}{*}{ Variables } & \multicolumn{5}{|c|}{ Disease-free survival $^{\mathrm{a}}$} & \multicolumn{5}{|c|}{ Overall survival ${ }^{\mathrm{b}}$} \\
\hline & \multirow[t]{2}{*}{$\mathrm{n}^{\mathrm{C}}$} & \multicolumn{2}{|c|}{ Recurrence } & \multirow[t]{2}{*}{$H R(95 \% C I)$} & \multirow[t]{2}{*}{ P-value ${ }^{\mathrm{d}}$} & \multirow[t]{2}{*}{$\mathrm{n}^{\mathrm{c}}$} & \multicolumn{2}{|c|}{ Death } & \multirow[t]{2}{*}{$H R(95 \% C I)$} & \multirow[t]{2}{*}{ P-value ${ }^{\mathrm{d}}$} \\
\hline & & Yes & No & & & & Yes & No & & \\
\hline \multicolumn{11}{|l|}{ Stage } \\
\hline I & 19 & 4 & 15 & Ref. & \multirow{4}{*}{0.004} & 19 & 3 & 16 & Ref. & \multirow{4}{*}{0.001} \\
\hline II & 8 & 3 & 5 & $2.0(0.5-9.1)$ & & 8 & 4 & 4 & $7.5(1.4-38.7)$ & \\
\hline III & 12 & 5 & 7 & $2.1(0.5-8.6)$ & & 12 & 6 & 6 & $6.8(1.3-36.2)$ & \\
\hline IV & 5 & 4 & 1 & $10.0(2.3-43.2)$ & & 5 & 4 & 1 & $18.7(3.4-104.2)$ & \\
\hline \multicolumn{11}{|c|}{ Vascular invasion } \\
\hline No & 13 & 3 & 10 & Ref. & \multirow[t]{2}{*}{0.07} & 16 & 5 & 11 & Ref. & \multirow[t]{2}{*}{0.015} \\
\hline Yes & 20 & 11 & 9 & $3.1(0.9-11.2)$ & & 25 & 15 & 10 & $3.4(1.2-9.6)$ & \\
\hline \multicolumn{11}{|c|}{ RASSF2 meth } \\
\hline No & 18 & 3 & 15 & Ref. & \multirow[t]{2}{*}{0.07} & 20 & 5 & 15 & Ref. & \multirow[t]{2}{*}{0.019} \\
\hline Yes & 28 & 12 & 16 & $2.9(0.8-10.3)$ & & 38 & 24 & 14 & $3.0(1.1-7.9)$ & \\
\hline
\end{tabular}

Abbreviations: CI, confidence interval; HR, hazard ratio; meth, methylation; Ref., reference value.

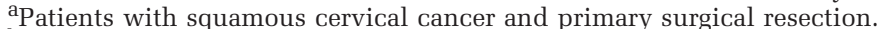

${ }^{\mathrm{b}}$ All the patients with squamous cervical cancer.

${ }^{\mathrm{C}}$ Case number (with available data).

${ }^{\mathrm{d}}$ Log-rank test value (statistically significant $P$-values in bold). 

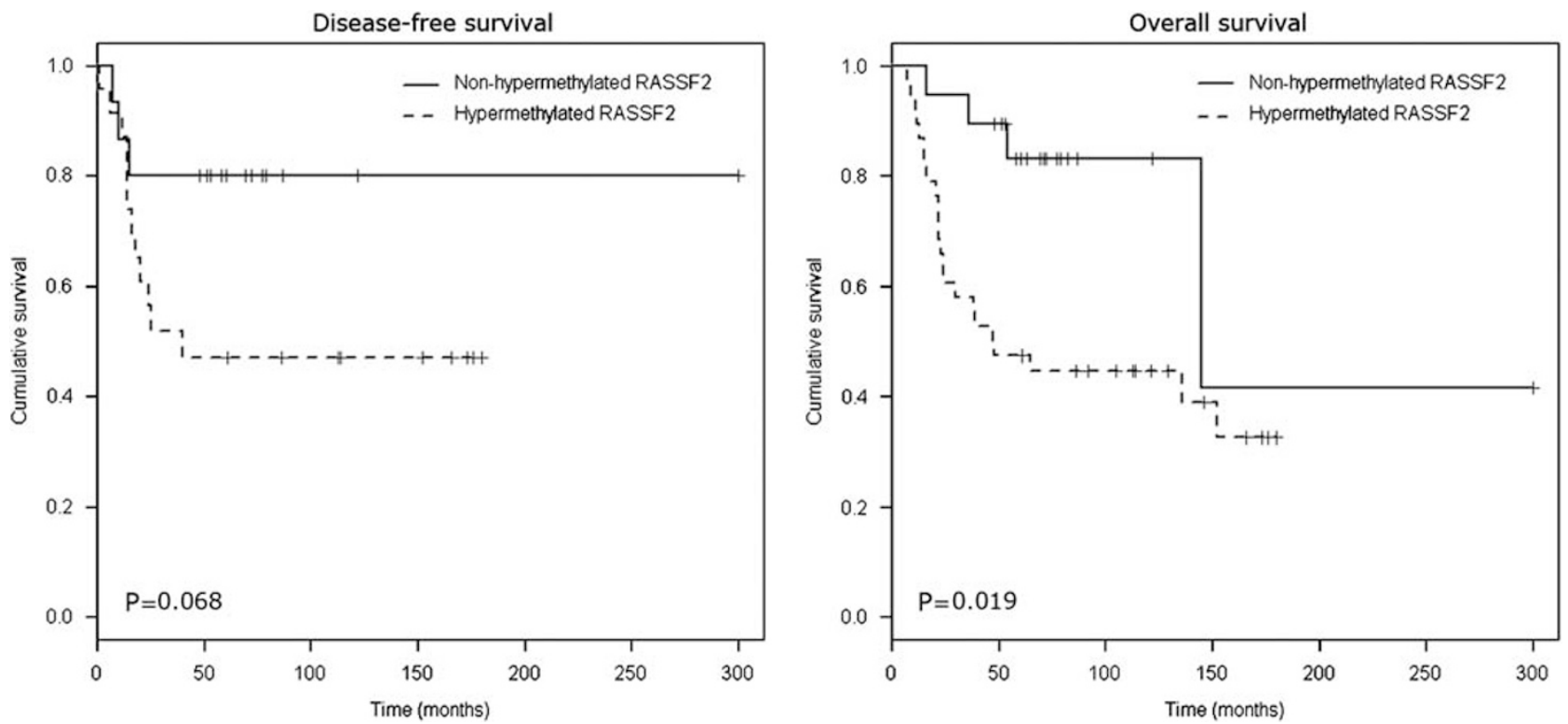

Figure 5 Kaplan-Meier plots stratified for RASSF2 promoter hypermethylation. (a) Curves for disease-free survival in patients with invasive carcinoma undergoing primary surgery. (b) Curves for overall survival in all squamous cervical carcinoma patients (primary surgical resection and biopsy).

Table 4 Cox proportional analysis of the risk of recurrence or death related to pathological and molecular variables in patients with squamous cervical cancer

\begin{tabular}{|c|c|c|c|c|}
\hline \multirow[t]{2}{*}{ Variable } & \multicolumn{2}{|c|}{ Disease-free survival $^{\text {a }}$} & \multicolumn{2}{|c|}{ Overall survival ${ }^{\mathrm{b}}$} \\
\hline & $H R(95 \% C I)$ & $\mathrm{P}$-value $\mathrm{c}$ & $H R(95 \% C I)$ & $\mathrm{P}$-value \\
\hline \multicolumn{5}{|c|}{ RASSF2A meth } \\
\hline No & Ref. & 0.03 & Ref. & 0.012 \\
\hline Yes & $6.2(1.2-32.4)$ & & $6.0(1.5-24.5)$ & \\
\hline \multicolumn{5}{|l|}{ Stage } \\
\hline I & Ref. & & Ref. & \\
\hline II & $2.6(0.46-14.7)$ & 0.06 & $5.8(1-32.6)$ & 0.005 \\
\hline III & $5.6(1-31.4)$ & & $21.3(3.8-118.6)$ & \\
\hline IV & $9.9(1.8-53.9)$ & & $20.6(3.2-133.9)$ & \\
\hline Age (years) & $1.039(0.98-1.1)$ & 0.17 & $1.038(0.99-1.1)$ & 0.13 \\
\hline
\end{tabular}

Abbreviations: CI, confidence interval; HR, hazard ratio; meth, methylation; Ref., reference value.

apatients with squamous cervical cancer and primary surgical resection.

${ }^{\mathrm{b}}$ All the patients with squamous cervical cancer.

${ }^{\mathrm{C}} P$-value (statistically significant values in bold).

to growth suppression and induction of apoptosis are also crucial for the progression of ovarian and lung cancers. ${ }^{45,46}$ In this study, we also found reexpression of this gene in SiHa cells after adding demethylating agents, demonstrating the role of hypermethylation in silencing this gene in cervical cancer. However, it was not associated with the clinicopathological variables included here.

In conclusion, we describe for the first time the preferential hypermethylation of the RASSF2 gene in squamous cervical carcinomas relative to precursor cervical intraepithelial neoplasia and also the association of this alteration with vascular invasion.
The hypermethylation of RASSF1 and EPB $41 L 3$ genes involved in cell signaling and cell adhesion, respectively, was also more frequent in squamous cervical cancer, although they were not of prognostic significance. Conversely, RASSF2 hypermethylation had a bad prognostic role in our study, which could be important to consider a stricter follow-up and treatment of these patients.

\section{Acknowledgements}

We are grateful to all the patients and clinicians who participated in the study. We also thank Dr X Bosch from the Cancer Epidemiology Research Program, Institut Català d'Oncologia (ICO, Barcelona, Spain), E Barba, F Garcia-Bragado, E Gochi, I Jauregui, B Larrea, J Perez and A Puras from Navarra Health Service (Pamplona, Spain), and V Coca, C Gorria, B Ibañez and E Reta from Navarrabiomed-Miguel Servet Foundation (Pamplona, Spain) for their technical assistance. This work was supported by two grants from the Department of Health of the Government of Navarra (Spain).

\section{Disclosure/conflict of interest}

The authors declare no conflict of interest.

\section{References}

1 Stat bite: cervical cancer mortality worldwide. J Natl Cancer Inst 2006;98:434.

2 Clifford GM, Smith JS, Plummer M, et al. Human papillomavirus types in invasive cervical cancer 
worldwide: a meta-analysis. Br J Cancer 2003;88: 63-73.

3 Wells M, Ostor AG, Crum CP, et al. Tumours of the uterine cervix: epithelial tumours, in World Health Organization Classification of Tumours, In: Tavassoli FA, Devilee P(eds). Pathology and Genetics of Tumours of the Breast and Female Genital Organs. The International Agency for Research on Cancer Press: Lyon, France; 2003, pp 259-289.

4 Kjaer SK, Frederiksen K, Munk C, et al. Long-term absolute risk of cervical intraepithelial neoplasia grade 3 or worse following human papillomavirus infection: role of persistence. J Natl Cancer Inst 2010;102: 1478-1488.

5 Esteller M. Epigenetics in cancer. $N$ Engl J Med 2008;358:1148-1159.

6 Cooper WN, Hesson LB, Matallanas D, et al. RASSF2 associates with and stabilizes the proapoptotic kinase MST2. Oncogene 2009;28:2988-2998.

7 Richter AM, Pfeifer GP, Dammann RH. The RASSF proteins in cancer; from epigenetic silencing to functional characterization. Biochim Biophys Acta 2009;1796:114-128.

8 Vos MD, Ellis CA, Elam C, et al. RASSF2 is a novel K-Ras-specific effector and potential tumor suppressor. J Biol Chem 2003;278:28045-28051.

9 Maruyama R, Akino K, Toyota M, et al. Cytoplasmic RASSF2A is a proapoptotic mediator whose expression is epigenetically silenced in gastric cancer. Carcinogenesis 2008;29:1312-1318.

10 Cooper WN, Dickinson RE, Dallol A, et al. Epigenetic regulation of the ras effector/tumour suppressor RASSF2 in breast and lung cancer. Oncogene 2008;27:1805-1811.

11 Hesson LB, Wilson R, Morton D, et al. CpG island promoter hypermethylation of a novel Ras-effector gene RASSF2A is an early event in colon carcinogenesis and correlates inversely with K-ras mutations. Oncogene 2005;24:3987-3994.

12 Liao X, Siu MK, Chan KY, et al. Hypermethylation of RAS effector related genes and DNA methyltransferase 1 expression in endometrial carcinogenesis. Int J Cancer 2008;123:296-302.

13 Schagdarsurengin U, Richter AM, Hornung J, et al. Frequent epigenetic inactivation of RASSF2 in thyroid cancer and functional consequences. Mol Cancer 2010;9:p 264.

14 Vos MD, Ellis CA, Elam C, et al. Ras uses the novel tumor suppressor RASSF1 as an effector to mediate apoptosis. J Biol Chem 2000;275:35669-35672.

15 Mitra S, Mazumder Indra D, Basu PS, et al. Alterations of RASSF1A in premalignant cervical lesions: clinical and prognostic significance. Mol Carcinogen 2012;51:723-733.

16 Huang KH, Huang SF, Chen IH, et al. Methylation of RASSF1A, RASSF2A, and HIN-1 is associated with poor outcome after radiotherapy, but not surgery, in oral squamous cell carcinoma. Clin Cancer Res 2009;15:4174-4180.

17 Eijsink JJ, Lendvai A, Deregowski V, et al. A four-gene methylation marker panel as triage test in high-risk human papillomavirus positive patients. Int J Cancer 2012;130:1861-1869.

18 Heller G, Geradts J, Ziegler B, et al. Downregulation of TSLC1 and DAL-1 expression occurs frequently in breast cancer. Breast Cancer Res Treat 2007;103: 283-291.
19 Yamada KS, Sakurai-Yageta WY, Masuda M, et al. Promoter hypermethylation of the potential tumor suppressor DAL-1/4.1B gene in renal clear cell carcinoma. Int J Cancer 2006;118:916-923.

20 Kikuchi S, Yamada D, Fukami T, et al. Promoter methylation of DAL-1/4.1B predicts poor prognosis in non-small cell lung cancer. Clin Cancer Res 2005;11:2954-2961.

21 Haie-Meder C, Maurice P, Castiglione M. ESMO Guidelines Working Group. Cervical cancer: ESMO Clinical Practice Guidelines for diagnosis, treatment and follow-up. Ann Oncol 2010;21:37-40.

22 Nonogaki S, Wakamatsu A, Filho AL, et al. Molecular strategies for identifying human papillomavirus infection in routinely processed samples: focus on paraffin sections. J Low Genit Tract Dis 2005;9: 219-224.

23 Van den Brule AJ, Pol R, Fransen-Daalmeijer N, et al. GP5 + /6 + PCR followed by reverse line blot analysis enables rapid and high-throughput identification of human papillomavirus genotypes. J Clin Microbiol 2002;40:779-787.

24 Herman JG, Graff JR, Myohanen S, et al. Methylationspecific PCR: a novel PCR assay for methylation status of CpG islands. Proc Natl Acad Sci USA 1996;93: 9821-9826.

25 Guerrero D, Guarch R, Ojer A, et al. Hypermethylation of the thrombospondin-1 gene is associated with poor prognosis in penile squamous cell carcinoma. BJU International 2008;102:747-755.

26 Luo D, Ye T, Li TQ, et al. Ectopic expression of RASSF2 and its prognostic role for gastric adenocarcinoma patients. Exp Ther Med 2012;3:391-396.

27 de Kok IM, van der Aa MA, van Ballegooijen M, et al. Trends in cervical cancer in the Netherlands until 2007: Has the bottom been reached? Int J Cancer 2010;128:2174-2181.

28 Henken FE, Wilting SM, Overmeer RM, et al. Sequential gene promoter methylation during HPV-induced cervical carcinogenesis. Br J Cancer 2007;97: 1457-1464.

29 Katargin AN, Pavlova LS, Kisseljov FL, et al. Hypermethylation of genomic $3.3-\mathrm{kb}$ repeats is frequent event in HPV-positive cervical cancer. BMC Med Genom 2009;2:30.

30 de Wilde J, Kooter JM, Overmeer RM, et al. hTERT promoter activity and CpG methylation in HPVinduced carcinogenesis. BMC Cancer 2010;10:271.

31 Virmani AK, Muller C, Rathi A, et al. Aberrant methylation during cervical carcinogenesis. Clin Cancer Res 2001;7:584-589.

32 Lee BB, Lee EJ, Jung EH, et al. Aberrant methylation of APC, MGMT, RASSF2A, and Wif-1 genes in plasma as a biomarker for early detection of colorectal cancer. Clin Cancer Res 2009;15:6185-6191.

33 Veena MS, Lee G, Keppler D, et al. Inactivation of the cystatin E/M tumor suppressor gene in cervical cancer. Genes Chromosomes Cancer 2008;47:740-754.

34 Yoshida S, Kajitani N, Satsuka A, et al. Ras modifies proliferation and invasiveness of cells expressing human papillomavirus oncoproteins. J Virol 2008;82:8820-8827.

35 Ren J, He W, Zhang R, et al. RASSF2A promoter methylation in hepatitis B virus-related hepatocellular carcinogenesis and its correlation with elevated serum alpha-fetoprotein level. J Huazhong Univ Sci Technolog Med Sci 2009;29:309-312. 
36 Guerrero D, Guarch R, Ojer A, et al. Differential hypermethylation of genes in vulvar cancer and lichen sclerosus coexisting or not with vulvar cancer. Int J Cancer 2011;128:2853-2864.

37 Biewenga P, van der Velden J, Mol BW, et al. Prognostic model for survival in patients with early stage cervical cancer. Cancer 2011;117:768-776.

38 Zhao L, Cui Q, Lu Z, et al. Aberrant methylation of RASSF2A in human pancreatic ductal adenocarcinoma and its relation to clinicopathologic features. Pancreas 2012;41:206-211.

39 Shi J, Zhang G, Yao D, et al. Prognostic significance of aberrant gene methylation in gastric cancer. Am J Cancer Res 2012;2:116-129.

40 Hiraki M, Kitajima Y, Koga Y, et al. Aberrant gene methylation is a biomarker for the detection of cancer cells in peritoneal wash samples from advanced gastric cancer patients. Ann Surg Oncol 2011;18: 3013-3019.

41 Lai HC, Lin YW, Chang CC, et al. Hypermethylation of two consecutive tumor suppressor genes, BLU and RASSF1A, located at 3p21.3 in cervical neoplasias. Gynecol Oncol 2007;104:629-635.
42 Lof-Ohlin ZM, Sorbe B, Wingren S, et al. Hypermethylation of promoter regions of the APC1A and p16INK4a genes in relation to prognosis and tumor characteristics in cervical cancer patients. Int $\mathrm{J}$ Oncol 2011;39:683-688.

43 Yang N, Eijsink JJ, Lendvai A, et al. Methylation markers for CCNA1 and C13ORF18 are strongly associated with high-grade cervical intraepithelial neoplasia and cervical cancer in cervical scrapings. Cancer Epidemiol Biomarkers Prev 2009;18: 3000-3007.

44 Choi YL, Kang SY, Shin YK, et al. Aberrant hypermethylation of RASSF1A promoter in ovarian borderline tumors and carcinomas. Virchows Arch 2006:448:331-336.

45 Yageta M, Kuramochi M, Masuda M, et al. Direct association of TSLC1 and DAL-1, two distinct tumor suppressor proteins in lung cancer. Cancer Res 2002;62:5129-5133.

46 Dafou D, Grun B, Sinclair J, et al. Microcell-mediated chromosome transfer identifies EPB41L3 as a functional suppressor of epithelial ovarian cancers. Neoplasia 2010;12:579-589.

Supplementary Information accompanies the paper on Modern Pathology website (http://www.nature.com/ modpathol) 